\title{
1 Firmicutes and Bacteroidetes explain mass gain variation in an obligate hibernator
}

2 Gina C. Johnson ${ }^{1}$, Samuel Degregori ${ }^{1}$, Paul H. Barber ${ }^{1}$ \& Daniel T. Blumstein ${ }^{1,2}$

$3 \quad{ }^{1}$ Department of Ecology and Evolutionary Biology, University of California, 621 Young Drive

4 South, Los Angeles, CA 90095-1606, USA

52 The Rocky Mountain Biological Laboratory, Box 519, Crested Butte, CO 81224, USA

6 Keywords: body condition, fitness, hibernation, life history, microbiome

\section{Abstract}

8 1. Body condition is an important life history challenge that directly impacts individual fitness and is particularly important for hibernating animals, whose maintenance of adequate body fat and mass is essential for survival.

2. It is well documented that symbiotic microorganisms play a vital role in animal physiology and behaviour. Recent work demonstrates that gut microbes are associated with fat accumulation and obesity; Firmicutes is consistently associated with obesity while Bacteroidetes is associated with leanness both in humans and other animals.

3. The focus of most microbiome studies has been on human health or involved lab reared animals used as a model system. However, these microbes likely are important for individual fitness in wild populations and provide potential mechanistic insights into the adaptability and survival of wildlife.

4. Here we test whether symbiotic microorganisms within the phyla of Firmicutes and Bacteroidetes are associated with summer mass gain in an exceptionally well-studied wild population of yellow-bellied marmots (Marmota flaviventer) by quantifying microbial abundance over five years of fecal samples (2015 - 2019) collected during their summer active season. 
5. Results show that marmots with higher mass gain rates have a greater abundance of Firmicutes. In contrast, higher abundance of Bacteroidetes was associated with lower mass gain rates, but only for marmots living in harsher environments. Similar patterns were found at the family level where Ruminococcaceae, a member of Firmicutes, was associated with higher mass gain rates, and Muribaculaceae, a member of Bacteroidetes, was associated with lower mass gain rates, and similarly in harsher environments.

6. Although correlative, these results highlight the importance of symbiotic gut microbiota

\section{Introduction}

34 The maintenance of sufficient body condition is a major life history challenge shared by many

35 animals with important implications for individual fitness (Gaillard et al., 2000; Green, 2001;

36 Schulte-Hostedde et al., 2001). Animals in good condition can endure longer fasting periods

37 (Atkinson \& Ramsay, 1995), are more likely to survive long migrations (Merilä \& Svensson,

38 1997), maintain a more responsive immune system (Navarro et al., 2003), have increased

39 fecundity (Tammaru et al., 1996) and enjoy higher mating success (Cotton et al., 2006).

One important measure of body condition affecting individual survival in many taxa is

41 relative body mass (Jakob et al., 1996; Schulte-Hostedde et al., 2001). For instance, larger body

42 mass increases the probability of survival in bighorn sheep (Ovis canadensis) (Festa-Bianchet et

43 al., 1997). Similarly, heavier canvasbacks (Aythya valisineria) have greater overwinter and

44 annual survival probabilities (Haramis et al., 1986). In contrast, great tits (Parus major) reduced

45 body mass in the presence of sparrowhawks (Accipiter nisus), reducing predation risk (Gosler 46 et al., 1995). 
While a variety of factors such as food availability, predation risk, and temperature influence individual body mass (Lima, 1986), a growing body of literature suggests that symbiotic microorganisms, collectively referred to as "microbiomes", also play a key role in

50 shaping host physiology (Neish, 2009; Kinross et al. 2011; Hird, 2017). The complex network of

51 microbes that reside in the vertebrate gastrointestinal tract influences the host's metabolic

52 activity and affects numerous aspects of physiology, anatomy, and behaviour (Cryan \& Dinan,

53 2012; Nicholson et al., 2012). Research on humans and other animals suggests a strong link

54 between the intestinal microbiome and mass gain (Ley et al., 2006; Tsai \& Coyle, 2009; Million

55 et al., 2012), with shifts in the dominant phyla of gut bacteria leading to obesity (Ley et al., 2005,

56 2006; Turnbaugh et al. 2006, 2008; Ley, 2010).

Most gut bacteria belong to four major phyla: Firmicutes, Bacteroidetes, Proteobacteria, and Actinobacteria (Tilg \& Kaser, 2011), sometimes referred to as the "core microbiome"

59 (Turnbaugh et al. 2009; Hird et al., 2015). Studies in mammals suggest that shifts in relative

60 abundance with more Firmicutes and fewer Bacteroidetes is associated with fat accumulation

61 and potential for obesity; in contrast, weight loss and leanness are associated with higher relative

62 abundance of Bacteroidetes (Ley et al., 2005; Turnbaugh \& Gordon, 2009). The balance of

63 Firmicutes and Bacteroidetes in the gut is often described as a ratio,

64 Firmicutes/Bacteroidetes (F/B), with obese individuals having a higher F/B ratio (Eckburg et al.,

65 2005) and lean individuals a lower F/B ratio (Mathur \& Barlow, 2015; Koliada et al., 2017).

66 That relative abundance of Firmicutes and Bacteroidetes influences obesity/leanness suggests

67 that gut microbiomes can affect energy extraction from the diet (Ellekilde et al., 2014), with

68 strong implications for individual fitness, especially for animals whose survival depends on

69 developing and maintaining adequate fat stores. 
Developing fat stores is critical to hibernating animals, whose long-term survival and

71 growth depend on adequate body fat accumulation (Turbill et al., 2001). Hibernation involves

72 dramatic seasonal changes in individual food consumption, body mass, and energy expenditure

73 (Lyman \& Chatfield, 1955; Florant et al., 2004). Moreover, hibernation alters the composition

74 and diversity of gut microbial communities across a diversity of taxa, ranging from mammals

75 (Dill-McFarland et al., 2014; Sonoyama et al., 2009; Malinčiová et al., 2017) to amphibians

76 (Kohl \& Yahn, 2016; Weng et al., 2016), and reptiles (Tang et al., 2019), suggesting that gut

77 microbiota may have functional importance in hibernating animals (Carey \& Assadi-Porter,

78 2017). For example, in juvenile Arctic ground squirrels, increases in Bacteroidetes and

79 reductions of Firmicutes reduces individual adiposity (Stevenson, Duddleston, \& Buck, 2014;

80 Stevenson, Buck, \& Duddleston, 2014) showing that microbial composition affects fat

81 deposition. Similarly, there is variation in gut microbiomes during the hibernation and active

82 phases of brown bears, and inoculating germ free mice with bear summer microbiota increases

83 mice fat accumulation (Sommer et al., 2016).

Yellow-bellied marmots (Marmota flaviventer) are obligate hibernators that must

85 accumulate sufficient fat stores to survive a 6-8 month hibernation (Hall \& Kelson, 1959;

86 Armitage, 1998; Armitage et al., 2003). Marmots lose up to half of their body mass during

87 hibernation (Armitage et al., 1976), thus mass gain during the active season is essential for

88 survival. Moreover, adequate fat stores are essential for reproductive female marmots to give

89 birth, directly influencing individual reproductive fitness (Andersen et al., 1976). Environmental

90 conditions largely explain variation in mass gain in marmots (Maldonado-Chaparro et al., 2015),

91 but age, sex, diet, food availability, and body size can also play a role (Armitage et al., 1976,

92 2003). However, given seasonal shifts in gut microbiomes of other hibernating animals 
93 (Stevenson et al., 2014a; Stevenson et al., 2014b; Sommer et al., 2016) and that microbiome

94 composition can influence mass gain (Ley et al., 2005; Turnbaugh \& Gordon, 2009; Ellekilde et

95 al., 2014), it is possible that gut microbiome composition could influence the survival and

96 reproductive fitness of individual marmots.

97 We examined the association of symbiotic gut bacteria and mass gain rate in an

98 exceptionally well-studied wild population of yellow-bellied marmots. This population has been

99 continuously monitored since 1962, providing long-term data on mass gain during the active

100 season, overwinter and summer survival, and reproductive success. Data shows that age and sex

101 play a critical role in mass gain (Armitage, 2014), with factors like chronic stress and

102 spatiotemporal variation affecting individual survival (Ozgul et al., 2006; Wey et al., 2014). Here

103 we use $16 \mathrm{~S}$ microbial metabarcoding to examine microbial composition in free-living marmots

104 to estimate the relative effects of microbiome composition and environmental factors in

105 explaining variation in mass gain rates. Specifically, we test the hypothesis that Firmicutes

106 abundance is associated with greater mass gain while Bacteroidetes abundance is associated with

107 lower mass gain rates.

108 Materials \& Methods

109 Study species and site

110 We studied yellow-bellied marmots in and around the Rocky Mountain Biological Laboratory

111 (RMBL), located in the Upper East River Valley in Gothic, Colorado, U.S.A. $\left(38^{\circ} 77^{\prime} \mathrm{N}\right.$,

$\left.112106^{\circ} 59^{\prime} \mathrm{W}\right)$. We trapped marmots by placing Tomahawk-live traps near burrow entrances. After

113 capture, the marmots were transferred to cloth handling bags to measure their body mass (to the

114 nearest $10 \mathrm{~g}$ ), and to determine their sex and reproductive status (Blumstein et al., 2006). Each

115 marmot was given a set of unique ear-tag numbers and their dorsal pelage marked with Nyanzol 
116 fur dye for identification from afar. Faecal samples are easily collected throughout the season

117 when animals are live trapped. When faeces are found in traps, they are routinely collected in a

118 plastic bag, immediately put on ice, and subsequently frozen at $-20{ }^{\circ} \mathrm{C}$ within $2 \mathrm{~h}$ of collection.

119 Samples are then transported from the field on dry ice and stored at $-80{ }^{\circ} \mathrm{C}$ in the lab for long-

120 term preservation.

121 From a large archive of faecal samples, we selected paired samples from individual

122 females to capture variation within the active season, although for some individuals only a single

123 sample was available. We focused on females, because both overwinter survival and

124 reproduction the next year depends on body condition (Andersen et al., 1976). We collected

125 samples from 10 different colonies: 5 from higher elevation colonies (mean elevation 3,043 m)

126 and 5 lower elevation colonies (mean elevation 2,883 m), separated by a maximum horizontal

127 distance of $4.9 \mathrm{~km}$. Although there is only an average of $160 \mathrm{~m}$ in altitude between these sites,

128 the phenology of these locations differs substantially, resulting in emergence from hibernation

129 and mating approximately 2 weeks earlier in down-valley colonies (Blumstein, 2009).

130 We selected samples collected closest to 1 June and 15 August, dates that fall within the

131 period of linear mass gain during their active season (Heissenberger et al., 2020), although some

132 samples were collected as early as May and as late as September. To maximise statistical power

133 and test for consistency across time, we selected samples across a five year span (2015 - 2019),

134 yielding 207 total samples representing 71 individuals. We sampled multiple age groups, 25

135 juvenile, 67 yearlings, and 109 adults (Table 1) because each age class faces unique ecological

136 challenges (Armitage et al., 1976; Heissenberger et al., 2020). Juveniles emerge from their natal

137 burrow in late June early July and must rapidly gain mass and body size to survive their first

138 hibernation, despite not reaching full body size until their second year. Yearlings tend to show 
139 the largest change in mass as they gain fat to survive hibernation but also undergo somatic

140 growth to reach adult body size during their second summer active season. Adults (defined as

141 reproductively mature females in their third year of life or older) have typically reached full body

142 size so only need to accumulate sufficient fat stores during their summer active season (Armitage

143 et al., 1976).

144 Microbiome sample processing and sequencing

145 We isolated bacterial DNA from faecal samples with Qiagen Powersoil Extraction kits following

146 the manufacturer's protocol (Germantown, Maryland, USA). We generated 16S libraries using

147 the 515F (5'-GTGCCAGCMGCCGCGGTAA) and 806R (5'-GGACTACHVHHHTWTCTAAT)

148 primers targeting the V4 region of the 16S rRNA gene (Caporaso et al., 2011). Samples

149 underwent PCR, in triplicate 25 ul reactions, using a Qiagen Multiplex PCR kit with the

150 following thermocycler conditions: 1 cycle of $94{ }^{\circ} \mathrm{C}$ for $3 \mathrm{~min} ; 35$ cycles of $94{ }^{\circ} \mathrm{C}$ for $45 \mathrm{~s}, 50{ }^{\circ} \mathrm{C}$

151 for $60 \mathrm{~s}$ and $72{ }^{\circ} \mathrm{C}$ for $90 \mathrm{~s}$; and 1 cycle of $72{ }^{\circ} \mathrm{C}$ for $10 \mathrm{~min}$ (Thompson et al., 2017). We pooled

152 triplicate reactions after confirming amplification success through gel electrophoresis, and then

153 dual-indexed samples using the Nextera UD Index Kit (Ilumina, San Diego, USA) and then

154 purified with OMEGA Bio-Tek MagBind magnetic beads (Norcross, Georgia, USA). Laragen

155 (Culver City, California, USA) performed quantification and pooling to create libraries with

156 equimolar sample concentrations. Multiplexed libraries were paired-end sequenced (300 bp per

157 sequence) on an Ilumina Miseq v3 at Laragen. We carried negative controls from the DNA

158 extraction process and subsequent PCR's throughout sample processing and added these to the

159 final pooled library for sequencing. 
161 The resulting sequence libraries were run through the QIIME2 (v. 2019.9) microbiome data

162 science platform (Bolyen et al., 2018) for quality control, amplicon sequence variant (ASV)

163 taxonomy assignment, and community diversity analyses. Data were demultiplexed and denoised

164 using dada2 (Callahan et al., 2016) and merged into a feature table for analysis. We then rarefied

165 samples to a minimum sequencing depth of 1000 reads, all samples with fewer than 1000 reads

166 were excluded from analysis. ASVs were assigned taxonomy using a naive Bayes taxonomy

167 classifier trained on the SILVA database (Quast et al., 2013; Yilmaz et al., 2014; Glöckner et al.,

168 2017) with reference sequences clustering at 99\% similarity. ASVs with fewer than 5 reads were

169 pruned as well as ASVs occurring in less than $3 \%$ of the samples (Karstens et al., 2019). Any

170 ASVs associated with assignments to eukaryotes, chloroplasts, and cyanobacterial reads were

171 also pruned. ASVs were compiled into a table and analyzed in R version 3.5.1. (R Core Team,

172 2017) using the package phyloseq (McMurdie \& Holmes, 2013).

\section{Estimating mass gain rate}

174 Because marmot mass gain rates vary with age (Armitage et al., 1976), repeated measures of

175 body mass were taken for all individuals captured from $2015-2019$. Using methods from

176 Heissenberger et al. (2020), we used body mass at emergence from the natal burrow for

177 juveniles, predicted 1 June body mass for yearlings and adults, and 15 August body mass for all

178 ages, dates that reflect the bulk of the growing season for each respective age class. Predicted

179 values were calculated by fitting a linear mixed effects model on body mass measurements,

180 where individual identity, year, and site were included as random effects and colony, age and sex

181 were fixed effects. This permitted us to generate Best Linear Unbiased Predictions (BLUPS) for

182 predicting 1 June and 15 August body mass (Heissenberger et al., 2020; Maldonado-Chaparro et

183 al., 2015; Ozgul et al., 2010). We calculated juvenile growth rate as the difference from the 15 
184 August body mass and the mass at first natal emergence divided by the number of days between

185 them. For yearlings and adults, it was the difference between 15 August and 1 June masses

186 divided by the number of days between them $(76 \mathrm{~d})$.

187 Testing bacterial composition influence on mass gain.

188 We fitted linear mixed effects models (Bates et al., 2015) to explain variation in mass gain rates.

189 To account for limitations in sequencing and to control for spurious correlations, the phyla OTU

190 tables were transformed using the centered log ratio, or CLR (Aitchison, 1986; Gloor et al.,

191 2017). Models included the fixed effects of CLR transformed ASV counts assigned to

192 Bacteroidetes or Firmicutes, and subsequent families within those phyla (these continuous

193 variables were zero and centered), valley position, and age class, and the interactions between

194 bacterial phyla or family and valley position and bacterial phyla or family and age class. We

195 included year and marmot ID as random effects. We included valley position, because snow

196 melts later at the higher elevation sites and this potential for an effect of elevation on mass gain,

197 combined with later marmot emergence means that they live in a relatively harsher environment

198 with less time to gain mass (Vuren \& Armitage, 1991; Blumstein, 2009; Armitage, 2014). Non-

199 significant interactions were removed and the models refitted for interpretation (Engqvist, 2005).

200 We estimated the marginal and conditional $\mathrm{R}^{2}$ values using the package MuMIn (Bartoń, 2015).

201 Lastly, we estimated the relative amount of variation explained by the bacterial taxa by removing

202 either the bacterial taxa or the significant interaction between the bacterial taxa and another fixed

203 effect, and refitting the final model without it.

\section{Results}

205 We amplified 207 samples, yielding a total of 10,930,721 raw sequencing reads. After cleaning

206 and filtering, 2,449,899 reads remained and were merged into a feature table for analysis. Sample 
207 sequencing depth ranged from 27 reads to 71,502 reads. As such, we rarefied all samples to a

208 minimum depth of 1000 reads, excluding a total of 6 samples with fewer than 1000 reads from

209 subsequent analysis, leaving a total of 201 samples representing 71 individuals across five years

$210 \quad(2015-2019)$.

211 Bacterial taxonomic composition of the marmot gut microbiome

212 At the phylum level, Firmicutes dominated marmot gut microbiomes, averaging $61 \%$ abundance

213 across all samples. Bacteroidetes was the second most dominant group, averaging $29 \%$ followed

214 by Tenericutes with an average of 5.6\% across all samples. Actinobacteria, Proteobacteria, and

215 Verrucomicrobia occupied the rest of most samples; the presence of other groups was less than

$2161 \%$ (Figure 1A). Examining microbial abundance at the family level showed that

217 Ruminococcaceae was the most dominant with an average abundance of $35 \%$, followed by

218 Lachnospiraceae with 15\%, Muribaculaceae with 12\%, and Rikenellaceae with 8.6\% mean

219 abundance across all samples. Rikenellaceae, Bacteroidaceae, Christensenellaceae, Clostridiales

220 vadinBB60 group, Anaeroplasmataceae, and Erysipelotrichaceae occupied the rest of most

221 samples (Figure 1B). Ruminococcaceae and Lachnospiraceae families are members of the

222 phylum Firmicutes, while Muribaculaceae and Rikenellaceae families are part of the

223 Bacteroidetes phylum.

224 Gut microbes explain variation in marmot mass gain rates

225 Mass gain rates across all 71 individuals varied by age class. Adults gained mass at an average of $22615.29 \mathrm{~g} /$ day, yearlings $21.08 \mathrm{~g} / \mathrm{day}$, and juveniles $24.53 \mathrm{~g} / \mathrm{day}$. The distribution of mass gain rates

227 conformed to normal expectations $(W=0.99185 ; P=0.3223)$ and therefore was not transformed.

228 After controlling for variation explained by age class and valley position as fixed effects, and

229 year and individual identity as random effects, we found that abundance of Firmicutes was 
230 positively associated with variation in mass gain rates (Figure 2; Table 2; estimate $=$

$2310.645 \pm 0.237 \mathrm{sem} ; P=0.007$; estimated partial $\left.R^{2}=0.011\right)$. Bacteroidetes was negatively

232 associated with variation in mass gain rates although with the opposite relationship, and only in

233 up valley colonies (Figure 3; Table 3; estimate $=-0.974 \pm 0.432$ sem; $P=0.026$; estimated

234 partial $\left.R^{2}=0.039\right)$.

235 Age class was included as a fixed effect in each mixed model, and was significantly 236 associated with mass gain rates for both juveniles (Table 2 (Firmicutes); estimate = -

$2373.505 \pm 1.174 \mathrm{sem} ; P=0.002)($ Table 3 (Bacteroidetes); estimate $=-$

$2383.158 \pm 1.188 \mathrm{sem} ; P=0.006)$ and for yearlings (Table 2; estimate $=$

$\left.2394.499 \pm 0.681 \mathrm{sem} ; P=1.67 \times 10^{-10}\right)\left(\right.$ Table 3 ; estimate $\left.=4.380 \pm 0.679 \mathrm{sem} ; P=4.24 \times 10^{-10}\right)$.

240 Age class explained much of the variation in mass gain rates in both the Firmicutes model

241 (estimated partial $R^{2}=0.159$ ) and the Bacteroidetes model (estimated partial $R^{2}=0.153$ ). Adults

242 were used as the reference age class. Valley position did not explain variation in mass gain rates

243 in either of the two models (Table 2; estimate $=1.601 \pm 1.209$ sem; $P=0.155)($ Table 3 ;

244 estimate $=1.167 \pm 1.204 \mathrm{sem} ; P=0.296)$.

245 Further analysis at the family level revealed that abundance of Ruminococcaceae within

246 the phylum Firmicutes, and Muribaculaceae within the phylum Bacteroidetes significantly

247 explained variation in mass gain rates. After controlling for variation explained by age class, and

248 valley position as fixed effects and year and individual identity as random effects, we found that

249 abundance of Ruminococcaceae was positively associated with variation in mass gain rates

250 (Figure 3A ; Table 4 ; estimate $=0.528 \pm 1.496 \mathrm{sem} ; P=0.016$; estimated partial $R^{2}=0.017$ ).

251 Muribaculaceae was negatively associated with variation in mass gain rates although with the

252 opposite relationship, and only in up valley colonies (Figure 3B ; Table 5 ; estimate = - 
254 final models by plotting their residuals (they were approximately normal), plotting q-q plots

255 (they were roughly straight), and plotting residuals vs. fitted values (there were no obvious

256 patterns).

Age class was also associated with mass gain rates at the family level analysis for both

258 juveniles (Table 4 (Ruminococcaceae); estimate $\left.=5.835 \pm 1.177 \mathrm{sem} ; P=1.68 \times 10^{-6}\right)($ Table 5

259 (Muribaculaceae); estimate $\left.=6.098 \pm 1.176 \mathrm{sem} ; P=6.02 \times 10^{-7}\right)$ and for yearlings $($ Table 4;

260 estimate $\left.=4.284 \pm 0.680 \mathrm{sem} ; P=2.00 \times 10^{-9}\right)($ Table $5 ;$ estimate $=4.357 \pm 0.676 \mathrm{sem} ; P=8.88$

$\left.261 \times 10^{-10}\right)$. Age class also explained much of the variation in mass gain rates in both the

262 Ruminococcaceae model (estimated partial $R^{2}=0.155$ ) and the Muribaculaceae model

263 (estimated partial $R^{2}=0.157$ ). Adults were used as the reference age class. Valley position did

264 not explain variation in mass gain rates in either of the two models (Table 4; estimate $=$

$2651.265 \pm 1.216 \mathrm{sem} ; P=0.301)($ Table 5; estimate $=1.123 \pm 1.204 \mathrm{sem} ; P=0.353)$.

266 Microbial composition is not influenced by age class or valley position

267 Individuals within the same age class or living in the same part of the valley (either at higher or

268 lower elevation sites) did not cluster by gut microbiome composition similarities. Principle-

269 coordinate analysis (PCoA) of Bray-Curtis distance metrics on the overall composition of the

270 marmot fecal microbiome revealed no pattern of clustering between different age classes or

271 individuals living up or down valley. PERMANOVA analysis confirmed no significant pattern

272 across age classes $(P=0.162)$ or valley position $(P=0.490)$ (Figure $\mathrm{S} 1)$.

\section{Discussion}

274 Comparison of microbiome composition shows that relative abundance of Firmicutes and

275 Bacteroidetes was significantly associated with mass gain in marmots, as reported for other 
mammals (Ley et al., 2005; Turnbaugh \& Gordon, 2009). Other studies on microbiomes and

277 mass gain remove animals from their native habitat, potentially altering the microbiome due to

278 changes in diet, environmental factors, and interactions with humans during captivity (Uenishi et

279 al., 2007; Dhanasiri et al., 2011; Clayton et al., 2016). Our study is unique in that we demonstrate

280 the effects of microbiomes on mass gain in wild, free-living population.

To our knowledge, this is the first study to investigate the effect of gut microbes on mass

282 gain in a hibernating animal from a wild population. Previous studies on hibernating species

283 found no significant effect of gut microbe abundance on seasonal fattening (Stevenson,

284 Duddleston, \& Buck, 2014; Sommer et al., 2016), a result that may result from low sample sizes

$285(N=46$ and $N=16)$ necessitated by keeping animals in captivity. By examining wild

286 populations, our study had a much larger sample size $(N=201)$, increasing the statistical power

287 to detect the subtle, but significant impact of microbiome composition on mass gain.

within those phyla, Ruminococcaceae and Muribaculaceae, was comparatively small compared

290 to other factors like age. However, this small effect size may be biologically consequential, as

291 even a few days variation in emergence time can be the difference between survival and death

292 (Armitage, 1976; Van Vuren \& Armitage, 1991). Further investigation is needed to determine

293 direct effects of microbes on survival. While age is a major factor (Armitage, 2014), valley

294 position and environmental conditions (Vuren \& Armitage, 1991) also explain variation in over-

295 winter survival. Given our prior research on marmots that have shown age and sex explain much

296 of the variation in mass gain and ability to fatten prior to hibernation, we expected the effects of

297 microbes to be relatively small. 
Like previous studies (Ley et al., 2005; Turnbaugh \& Gordon, 2009), our results from marmots showed that relative abundance of Firmicutes and Bacteroidetes had a significant impact on mass gain. However, we were able to show that these patterns largely resulted from the abundance of Ruminococcaceae and Muribaculaceae. Specifically, animals with higher mass gain rates had a higher abundance of Ruminococcaceae (a Firmicutes), while animals with lower mass gain rates had a higher abundance of Muribaculaceae (a Bacteroidetes). The latter result, however, was only true in up-valley colonies where winter conditions are harsher. While an animals' environment can directly influence phenotype, such as white coats in coyotes and snowshoe hares during winter months, bacterial symbionts also influence phenotype (Lynch \& Hsiao, 2019). Therefore, the host - microbiome - environment relationship can be 308 complex and vary across individuals that live in different places (Koskella \& Bergelson, 2020).

309 In this study, the association of Bacteroidetes/Muribaculaceae was associated with lower mass

310 gain rates only in animals from higher elevation colonies. It could be that marmots that live in

311 lower elevation and less harsh conditions are less likely to be influenced by variation in

312 Bacteroidetes because they are able to offset the cost of having more Bacteroidetes by eating and

313 gaining mass for a longer period of time. Snow melts at our lower elevation sites about two

314 weeks earlier than our higher elevation sites leading to an extended growing season (Van Vuren

315 \& Armitage, 1991; Blumstein, 2009; Armitage, 2014). Therefore, animals living in harsher

316 environments may be more effected by the Bacteroidetes than those living in less harsh

317 conditions.

318 Interestingly, our results show no patterns of similarities or clustering between the 319 different age classes or animals living at different elevations. Lack of clustering between age 320 classes may be due to the social behavior of this species (Armitage, 1991), because regardless of 
321 age, all animals in groups within colonies are sharing the same burrow and consistently

322 interacting with one another (Blumstein et al. 2004) and social behavior has been shown to be a

323 direct mode for microbial transmission (Archie \& Tung, 2015; Sarkar et al. 2020). Additionally,

324 studies have shown social species to share similar microbiomes with individuals whom they have

325 the most interactions (Moeller et al. 2016). Lack of clustering by colony elevation may be

326 explained by similar diets in each region. The furthest distance between up and down-valley

327 colonies is $4.9 \mathrm{~km}$ and vegetation types are essentially identical. It is expected that with

328 increasing physical distance between hosts, $\beta$-diversity between hosts or groups would increase

329 because microbial transmission is attenuated (Moeller et al. 2017). Although dispersal between

330 these valley areas is rare, animals occupy the same valley, and are therefore not geographically

331 separated (Armitage, 1991).

Our results are consistent with many human studies that show an association between

333 Firmicutes and obesity, while leanness is associated with Bacteroidetes (Ley et al., 2005, 2006;

334 Abdallah Ismail et al., 2011; Koliada et al., 2017). Given that marmots are obligate hibernators

335 and fat accumulation is an indicator of good body condition and directly related to fitness in the

336 wild (Haramis et al., 1986; Tammaru et al., 1996; Merilä \& Svensson, 1997; Festa-Bianchet et

337 al., 1997), the gut microbiome composition of marmots appears to favor weight gain that should

338 confer greater survival and reproductive success (Armitage, 1991; Armitage, 1998). Although

339 our results show that relative abundance of Firmicutes and Bacteroidetes is associated with mass

340 gain rates in marmots, these results are only correlative. Further, analyses at the family level

341 indicates selected taxa are likely involved in mass accumulation. Experimental studies similar to

342 Stevenson, Buck, \& Duddleston (2014), Stevenson, Duddleston, \& Buck (2014), and Sommer et

343 al. (2016), or multilevel structural equation modelling (Shipley, 2009) are needed to confirm 
344 causation, and to identify which bacteria are directly involved in increased mass gain rates and

345 end of season mass gain in yellow bellied marmots. Additionally, investigating modes of

346 microbial transmission, whether it be through social interactions, diet, or the environment could

347 help determine why certain microbe species would be present in some individuals and not others.

348 The host-microbiota symbiosis is likely an important component to the hibernation

349 phenotype. Overall, understanding the role gut microbes play in life history traits will be

350 valuable for long-term research studies that aim to understand the implications for individual

351 fitness. This study is novel because the majority of studies that examine the relationship between

352 hosts and their resident microbes have been limited to humans, lab reared animals, and captive

353 animals. Thus, studies which examine natural variation in the wild are needed if we are to

354 understand how important these effects are in nature, and if we are to conduct robust

355 comparative studies (Hird, 2017). Marmots are an excellent species to study to understand how

356 microbes may impact fitness because they live in harsh, seasonal environments, however, studies

357 on other species are needed. Gaining insight on how animals are affected by their resident

358 microbes can help us understand the unique adaptations to harsh conditions and a changing

359 dietary landscape and will provide essential information to future conservation and management

360 (Mueller et al., 2020), and applications for human and animal health. Symbiotic microbes are

361 important to animals and humans alike, and investigating this relationship in an evolutionary

362 context is of great interest and importance.

\section{References}

365 Abdallah Ismail, N., Ragab, S. H., Abd ElBaky, A., Shoeib, A. R. S., Alhosary, Y., \& Fekry, D. 366 (2011). Frequency of Firmicutes and Bacteroidetes in gut microbiota in obese and normal weight 367 Egyptian children and adults. Archives of Medical Science : AMS, 7(3), 501-507.

368 https://doi.org/10.5114/aoms.2011.23418 
Aitchison, J. (1982). The Statistical Analysis of Compositional Data. Journal of the Royal

Statistical Society: Series B (Methodological), 44(2), 139-160. https://doi.org/10.1111/j.25176161.1982.tb01195.x

Andersen, D. C., Armitage, K. B., \& Hoffmann, R. S. (1976). Socioecology of Marmots: Female Reproductive Strategies. Ecology, 57(3), 552-560. https://doi.org/10.2307/1936439

Archie, E. A., \& Tung, J. (2015). Social behavior and the microbiome. Current Opinion in Behavioral Sciences, 6, 28-34. https://doi.org/10.1016/j.cobeha.2015.07.008

Armitage, K B. (1991). Social and Population Dynamics of Yellow-Bellied Marmots: Results from Long-Term Research. Annual Review of Ecology and Systematics, 22(1), 379-407. https://doi.org/10.1146/annurev.es.22.110191.002115

Armitage, K. B. (1998). Reproductive Strategies of Yellow-Bellied Marmots: Energy Conservation and Differences between the Sexes. Journal of Mammalogy, 79(2), 385-393. https://doi.org/10.2307/1382969

Armitage, K. B. (2014). Marmot Biology: Sociality, Individual Fitness, and Population Dynamics. Cambridge University Press.

Armitage, K. B, Blumstein, D. T., \& Woods, B. C. (2003). Energetics of hibernating yellowbellied marmots (Marmota flaviventris). Comparative Biochemistry and Physiology Part A: Molecular \& Integrative Physiology, 134(1), 101-114. https://doi.org/10.1016/S10956433(02)00219-2

Armitage, K. B., Downhower, J. F., \& Svendsen, G. E. (1976). Seasonal Changes in Weights of Marmots. The American Midland Naturalist, 96(1), 36-51. https://doi.org/10.2307/2424566

Atkinson, S. N., \& Ramsay, M. A. (1995). The Effects of Prolonged Fasting of the Body Composition and Reproductive Success of Female Polar Bears (Ursus maritimus). Functional Ecology, 9(4), 559-567. https://doi.org/10.2307/2390145

Bartoń, K. (2015). MuMIn: Model selection and model averaging based on information criteria (AICc and alike). (https://cran.r-project.org/web/packages/MuMIn/index.html)

Bates, D., Mächler, M., Bolker, B., \& Walker, S. (2014). Fitting Linear Mixed-Effects Models using lme4. ArXiv:1406.5823 [Stat]. http://arxiv.org/abs/1406.5823

Blumstein, D. T. (2009). Social Effects on Emergence from Hibernation in Yellow-Bellied Marmots. Journal of Mammalogy, 90(5), 1184-1187. https://doi.org/10.1644/08-MAMM-A344.1

Blumstein, D. T., Im, S., Nicodemus, A., \& Zugmeyer, C. (2004). Yellow-Bellied Marmots (Marmota flaviventris) Hibernate Socially. Journal of Mammalogy, 85(1), 25-29. https://doi.org/10.1644/1545-1542(2004)085<0025:YMMFHS>2.0.CO;2 
Blumstein, D. T., Ozgul, A., Yovovich, V., Vuren, D. H. V., \& Armitage, K. B. (2006). Effect of predation risk on the presence and persistence of yellow-bellied marmot (Marmota flaviventris) colonies. Journal of Zoology, 270(1), 132-138. https://doi.org/10.1111/j.14697998.2006.00098.x

Bolyen, E., Rideout, J. R., Dillon, M. R., Bokulich, N. A., Abnet, C., Al-Ghalith, G. A., Alexander, H., Alm, E. J., Arumugam, M., Asnicar, F., Bai, Y., Bisanz, J. E., Bittinger, K., Brejnrod, A., Brislawn, C. J., Brown, C. T., Callahan, B. J., Caraballo-Rodríguez, A. M., Chase, J., ... Caporaso, J. G. (2018). QIIME 2: Reproducible, interactive, scalable, and extensible microbiome data science [Preprint]. PeerJ Preprints.

https://doi.org/10.7287/peerj.preprints.27295v2

Callahan, B. J., McMurdie, P. J., Rosen, M. J., Han, A. W., Johnson, A. J. A., \& Holmes, S. P. (2016). DADA2: High-resolution sample inference from Illumina amplicon data. Nature Methods, 13(7), 581-583. https://doi.org/10.1038/nmeth.3869

Caporaso, J. G., Lauber, C. L., Walters, W. A., Berg-Lyons, D., Lozupone, C. A., Turnbaugh, P. J., Fierer, N., \& Knight, R. (2011). Global patterns of 16S rRNA diversity at a depth of millions of sequences per sample. Proceedings of the National Academy of Sciences, 108(Supplement 1), 4516-4522. https://doi.org/10.1073/pnas.1000080107

Carey, H. V., \& Assadi-Porter, F. M. (2017). The Hibernator Microbiome: Host-Bacterial Interactions in an Extreme Nutritional Symbiosis. Annual Review of Nutrition, 37(1), 477-500. https://doi.org/10.1146/annurev-nutr-071816-064740

Clayton, J. B., Vangay, P., Huang, H., Ward, T., Hillmann, B. M., Al-Ghalith, G. A., Travis, D. A., Long, H. T., Tuan, B. V., Minh, V. V., Cabana, F., Nadler, T., Toddes, B., Murphy, T., Glander, K. E., Johnson, T. J., \& Knights, D. (2016). Captivity humanizes the primate microbiome. Proceedings of the National Academy of Sciences, 113(37), 10376-10381. https://doi.org/10.1073/pnas.1521835113

Cotton, S., Small, J., \& Pomiankowski, A. (2006). Sexual Selection and Condition-Dependent Mate Preferences. Current Biology, 16(17), R755-R765. https://doi.org/10.1016/j.cub.2006.08.022

Cryan, J. F., \& Dinan, T. G. (2012). Mind-altering microorganisms: The impact of the gut microbiota on brain and behaviour. Nature Reviews Neuroscience, 13(10), 701-712. https://doi.org/10.1038/nrn3346

Dhanasiri, A. K. S., Brunvold, L., Brinchmann, M. F., Korsnes, K., Bergh, Ø., \& Kiron, V. (2011). Changes in the intestinal microbiota of wild Atlantic cod Gadus morhua L. upon captive rearing. Microbial Ecology, 61(1), 20-30. https://doi.org/10.1007/s00248-010-9673-y

Dill-McFarland, K. A., Neil, K. L., Zeng, A., Sprenger, R. J., Kurtz, C. C., Suen, G., \& Carey, H. V. (2014). Hibernation alters the diversity and composition of mucosa-associated bacteria while enhancing antimicrobial defence in the gut of 13-lined ground squirrels. Molecular Ecology, 
23(18), 4658-4669. https://doi.org/10.1111/mec.12884

Eckburg, P. B., Bik, E. M., Bernstein, C. N., Purdom, E., Dethlefsen, L., Sargent, M., Gill, S. R., Nelson, K. E., \& Relman, D. A. (2005). Diversity of the Human Intestinal Microbial Flora. Science, 308(5728), 1635-1638. https://doi.org/10.1126/science.1110591

Ellekilde, M., Selfjord, E., Larsen, C. S., Jakesevic, M., Rune, I., Tranberg, B., Vogensen, F. K., Nielsen, D. S., Bahl, M. I., Licht, T. R., Hansen, A. K., \& Hansen, C. H. F. (2014). Transfer of gut microbiota from lean and obese mice to antibiotic-treated mice. Scientific Reports, 4(1), 5922. https://doi.org/10.1038/srep05922

Engqvist, L. (2005). The mistreatment of covariate interaction terms in linear model analyses of behavioural and evolutionary ecology studies. Animal Behaviour, 70(4), 967-971. https://doi.org/10.1016/j.anbehav.2005.01.016

Festa-Bianchet, M., Jorgenson, J. T., Bérubé, C. H., Portier, C., \& Wishart, W. D. (2011). Body mass and survival of bighorn sheep. Canadian Journal of Zoology. https://doi.org/10.1139/z97$\underline{763}$

Florant, G. L., Porst, H., Peiffer, A., Hudachek, S. F., Pittman, C., Summers, S. A., Rajala, M. W., \& Scherer, P. E. (2004). Fat-cell mass, serum leptin and adiponectin changes during weight gain and loss in yellow-bellied marmots (Marmota flaviventris). Journal of Comparative Physiology. B, Biochemical, Systemic, and Environmental Physiology, 174(8), 633-639. https://doi.org/10.1007/s00360-004-0454-0

Gaillard, J.-M., Festa-Bianchet, M., Delorme, D., \& Jorgenson, J. (2000). Body mass and individual fitness in female ungulates: Bigger is not always better. Proceedings of the Royal Society of London. Series B: Biological Sciences, 267(1442), 471-477.

https://doi.org/10.1098/rspb.2000.1024

Glöckner, F. O., Yilmaz, P., Quast, C., Gerken, J., Beccati, A., Ciuprina, A., Bruns, G., Yarza, P., Peplies, J., Westram, R., \& Ludwig, W. (2017). 25 years of serving the community with ribosomal RNA gene reference databases and tools. Journal of Biotechnology, 261, 169-176. https://doi.org/10.1016/j.jbiotec.2017.06.1198

Gloor, G. B., Macklaim, J. M., Pawlowsky-Glahn, V., \& Egozcue, J. J. (2017). Microbiome Datasets Are Compositional: And This Is Not Optional. Frontiers in Microbiology, 8. https://doi.org/10.3389/fmicb.2017.02224

Green, A. J. (2001). Mass/Length Residuals: Measures of Body Condition or Generators of Spurious Results? Ecology, 82(5), 1473-1483. https://doi.org/10.1890/00129658(2001)082[1473:MLRMOB]2.0.CO;2

Hall, E., \& Kelson, K. R. (1959). The mammals of North America. 2 Vols. Ronald Press. https://tamug-ir.tdl.org/handle/1969.3/25462 
Haramis, G. M., Nichols, J. D., Pollock, K. H., \& Hines, J. E. (1986). The Relationship Between Body Mass and Survival of Wintering Canvasbacks. The Auk, 103(3), 506-514. https://doi.org/10.1093/auk/103.3.506

Heissenberger, S., de Pinho, G. M., Martin, J. G. A., \& Blumstein, D. T. (2020). Age and location influence the costs of compensatory and accelerated growth in a hibernating mammal. Behavioral Ecology, 31(3), 826-833. https://doi.org/10.1093/beheco/araa013

Hird, S. M. (2017). Evolutionary Biology Needs Wild Microbiomes. Frontiers in Microbiology, 8. https://doi.org/10.3389/fmicb.2017.00725

Hird, S. M., Sánchez, C., Carstens, B. C., \& Brumfield, R. T. (2015). Comparative Gut Microbiota of 59 Neotropical Bird Species. Frontiers in Microbiology, 6. https://doi.org/10.3389/fmicb.2015.01403

Jakob, E. M., Marshall, S. D., \& Uetz, G. W. (1996). Estimating Fitness: A Comparison of Body Condition Indices. Oikos, 77(1), 61-67. https://doi.org/10.2307/3545585

Karstens, L., Asquith, M., Davin, S., Fair, D., Gregory, W. T., Wolfe, A. J., Braun, J., \& McWeeney, S. (2019). Controlling for Contaminants in Low-Biomass 16S rRNA Gene Sequencing Experiments. MSystems, 4(4), Article 4. https://doi.org/10.1128/mSystems.00290-19

Kinross, J. M., Darzi, A. W., \& Nicholson, J. K. (2011). Gut microbiome-host interactions in health and disease. Genome Medicine, 3(3), 14. https://doi.org/10.1186/gm228

Kohl, K. D., \& Yahn, J. (2016). Effects of environmental temperature on the gut microbial communities of tadpoles. Environmental Microbiology, 18(5), 1561-1565. https://doi.org/10.1111/1462-2920.13255

Koliada, A., Syzenko, G., Moseiko, V., Budovska, L., Puchkov, K., Perederiy, V., Gavalko, Y., Dorofeyev, A., Romanenko, M., Tkach, S., Sineok, L., Lushchak, O., \& Vaiserman, A. (2017). Association between body mass index and Firmicutes/Bacteroidetes ratio in an adult Ukrainian population. BMC Microbiology, 17(1), 120. https://doi.org/10.1186/s12866-017-1027-1

Koskella, B., \& Bergelson, J. (2020). The study of host-microbiome (co)evolution across levels of selection. Philosophical Transactions of the Royal Society B: Biological Sciences, 375(1808), 20190604. https://doi.org/10.1098/rstb.2019.0604

Ley, R. E. (2010). Obesity and the human microbiome. Current Opinion in Gastroenterology, 26(1), 5-11. https://doi.org/10.1097/MOG.0b013e328333d751

Ley, R. E., Bäckhed, F., Turnbaugh, P., Lozupone, C. A., Knight, R. D., \& Gordon, J. I. (2005). Obesity alters gut microbial ecology. Proceedings of the National Academy of Sciences, 102(31), 11070-11075. https://doi.org/10.1073/pnas.0504978102

Ley, R. E., Turnbaugh, P. J., Klein, S., \& Gordon, J. I. (2006). Human gut microbes associated 
with obesity. Nature, 444(7122), 1022-1023. https://doi.org/10.1038/4441022a

Lima, S. L. (1986). Predation Risk and Unpredictable Feeding Conditions: Determinants of Body Mass in Birds. Ecology, 67(2), 377-385. https://doi.org/10.2307/1938580

Lyman, C. P., \& Chatfield, P. O. (1955). Physiology of Hibernation in Mammals. Physiological Reviews, 35(2), 403-425. https://doi.org/10.1152/physrev.1955.35.2.403

Lynch, J. B., \& Hsiao, E. Y. (2019). Microbiomes as sources of emergent host phenotypes. Science, 365(6460), 1405-1409. https://doi.org/10.1126/science.aay0240

Maldonado-Chaparro, A. A., Martin, J. G. A., Armitage, K. B., Oli, M. K., \& Blumstein, D. T. (2015). Environmentally induced phenotypic variation in wild yellow-bellied marmots. Journal of Mammalogy, 96(2), 269-278. https://doi.org/10.1093/jmammal/gyu006

Malinčiová, L., Hrehová, L., Maxinová, E., Uhrin, M., \& Pristaš, P. (2017). The dynamics of Mediterranean horseshoe bat (Rhinolophus euryale, Chiroptera) gut microflora during hibernation. Acta Chiropterologica, 19(1), 211-218. https://doi.org/10.3161/15081109ACC2017.19.1.017

Mathur, R., \& Barlow, G. M. (2015). Obesity and the microbiome. Expert Review of Gastroenterology \& Hepatology, 9(8), 1087-1099. https://doi.org/10.1586/17474124.2015.1051029

McMurdie, P. J., \& Holmes, S. (2013). phyloseq: An R Package for Reproducible Interactive Analysis and Graphics of Microbiome Census Data. PLOS ONE, 8(4), e61217. https://doi.org/10.1371/journal.pone.0061217

Merilä, J., \& Svensson, E. (1997). Are Fat Reserves in Migratory Birds Affected by Condition in Early Life? Journal of Avian Biology, 28(4), 279-286. https://doi.org/10.2307/3676940

Million, M., Angelakis, E., Paul, M., Armougom, F., Leibovici, L., \& Raoult, D. (2012). Comparative meta-analysis of the effect of Lactobacillus species on weight gain in humans and animals. Microbial Pathogenesis, 53(2), 100-108. https://doi.org/10.1016/j.micpath.2012.05.007

Moeller, A. H., Foerster, S., Wilson, M. L., Pusey, A. E., Hahn, B. H., \& Ochman, H. (2016). Social behavior shapes the chimpanzee pan-microbiome. Science Advances, 2(1), e1500997. https://doi.org/10.1126/sciadv.1500997

Moeller, A. H., Suzuki, T. A., Lin, D., Lacey, E. A., Wasser, S. K., \& Nachman, M. W. (2017). Dispersal limitation promotes the diversification of the mammalian gut microbiota. Proceedings of the National Academy of Sciences, 114(52), 13768-13773.

https://doi.org/10.1073/pnas.1700122114

Mueller, E. A., Wisnoski, N. I., Peralta, A. L., \& Lennon, J. T. (2020). Microbial rescue effects: How microbiomes can save hosts from extinction. Functional Ecology, 34(10), 2055-2064. 
https://doi.org/10.1111/1365-2435.13493

601

602

603

604

605

606

607

608

609

610

611

612

613

614

615

616

617

618

619

620

621

622

623

624

625

626

627

628

629

630

631

632

633

634

635

636

637

638

639

640

641

642

643

644

645
Navarro, C., Marzal, A., Lope, F. D., \& Møller, A. P. (2003). Dynamics of an immune response in house sparrows Passer domesticus in relation to time of day, body condition and blood parasite infection. Oikos, 101(2), 291-298. https://doi.org/10.1034/j.1600-0706.2003.11663.x

Neish, A. S. (2009). Microbes in Gastrointestinal Health and Disease. Gastroenterology, 136(1), 65-80. https://doi.org/10.1053/j.gastro.2008.10.080

Nicholson, J. K., Holmes, E., Kinross, J., Burcelin, R., Gibson, G., Jia, W., \& Pettersson, S. (2012). Host-Gut Microbiota Metabolic Interactions. Science, 336(6086), 1262-1267.

https://doi.org/10.1126/science.1223813

Ozgul, A., Armitage, K. B., Blumstein, D. T., \& Oli, M. K. (2006). Spatiotemporal Variation in Survival Rates: Implications for Population Dynamics of Yellow-Bellied Marmots. Ecology, 87(4), 1027-1037. https://doi.org/10.1890/0012-9658(2006)87[1027:SVISRI]2.0.CO;2

Ozgul, A., Childs, D. Z., Oli, M. K., Armitage, K. B., Blumstein, D. T., Olson, L. E., Tuljapurkar, S., \& Coulson, T. (2010). Coupled dynamics of body mass and population growth in response to environmental change. Nature, 466(7305), 482-485.

https://doi.org/10.1038/nature09210

Quast, C., Pruesse, E., Yilmaz, P., Gerken, J., Schweer, T., Yarza, P., Peplies, J., \& Glöckner, F. O. (2013). The SILVA ribosomal RNA gene database project: Improved data processing and web-based tools. Nucleic Acids Research, 41(Database issue), D590-D596.

https://doi.org/10.1093/nar/gks1219

R Core Team. (2014). R: a language and environment for statistical computing. Vienna: R Foundation for Statistical Computing. https://www.R-project.org/.

Sarkar, A., Harty, S., Johnson, K. V.-A., Moeller, A. H., Archie, E. A., Schell, L. D., Carmody, R. N., Clutton-Brock, T. H., Dunbar, R. I. M., \& Burnet, P. W. J. (2020a). Microbial transmission in animal social networks and the social microbiome. Nature Ecology \& Evolution, 4(8), 1020-1035. https://doi.org/10.1038/s41559-020-1220-8

Schulte-Hostedde, A. I., Millar, J. S., \& Hickling, G. J. (2011). Evaluating body condition in small mammals. Canadian Journal of Zoology. https://doi.org/10.1139/z01-073

Shipley, B. (2009). Confirmatory path analysis in a generalized multilevel context. Ecology, 90(2), 363-368. https://doi.org/10.1890/08-1034.1

Sommer, F., Ståhlman, M., Ilkayeva, O., Arnemo, J. M., Kindberg, J., Josefsson, J., Newgard, C. B., Fröbert, O., \& Bäckhed, F. (2016). The Gut Microbiota Modulates Energy Metabolism in the Hibernating Brown Bear Ursus arctos. Cell Reports, 14(7), 1655-1661.

https://doi.org/10.1016/j.celrep.2016.01.026 
Sonoyama, K., Fujiwara, R., Takemura, N., Ogasawara, T., Watanabe, J., Ito, H., \& Morita, T. (2009). Response of Gut Microbiota to Fasting and Hibernation in Syrian Hamsters. Applied and Environmental Microbiology, 75(20), 6451-6456. https://doi.org/10.1128/AEM.00692-09

Stevenson, T. J., Buck, C. L., \& Duddleston, K. N. (2014). Temporal Dynamics of the Cecal Gut Microbiota of Juvenile Arctic Ground Squirrels: A Strong Litter Effect across the First Active Season. Applied and Environmental Microbiology, 80(14), 4260-4268. https://doi.org/10.1128/AEM.00737-14

Stevenson, T. J., Duddleston, K. N., \& Buck, C. L. (2014). Effects of Season and Host Physiological State on the Diversity, Density, and Activity of the Arctic Ground Squirrel Cecal Microbiota. Applied and Environmental Microbiology, 80(18), 5611-5622. https://doi.org/10.1128/AEM.01537-14

\section{Tammaru, T., Kaitaniemi, P., \& Ruohomäki, K. (1996). Realized Fecundity in Epirrita} autumnata (Lepidoptera: Geometridae): Relation to Body Size and Consequences to Population Dynamics. Oikos, 77(3), 407-416. https://doi.org/10.2307/3545931

Tang, K.-Y., Wang, Z.-W., Wan, Q.-H., \& Fang, S.-G. (2019). Metagenomics Reveals Seasonal Functional Adaptation of the Gut Microbiome to Host Feeding and Fasting in the Chinese Alligator. Frontiers in Microbiology, 10. https://doi.org/10.3389/fmicb.2019.02409

Thompson, L. R., Sanders, J. G., McDonald, D., Amir, A., Ladau, J., Locey, K. J., Prill, R. J., Tripathi, A., Gibbons, S. M., Ackermann, G., Navas-Molina, J. A., Janssen, S., Kopylova, E., Vázquez-Baeza, Y., González, A., Morton, J. T., Mirarab, S., Zech Xu, Z., Jiang, L., ... Knight, R. (2017). A communal catalogue reveals Earth's multiscale microbial diversity. Nature, 551(7681), 457-463. https://doi.org/10.1038/nature24621

Tilg, H., \& Kaser, A. (2011). Gut microbiome, obesity, and metabolic dysfunction. The Journal of Clinical Investigation, 121(6), 2126-2132. https://doi.org/10.1172/JCI58109

Tsai, F., \& Coyle, W. J. (2009). The microbiome and obesity: Is obesity linked to our gut flora? Current Gastroenterology Reports, 11(4), 307-313. https://doi.org/10.1007/s11894-009-0045-z

Turbill, C., Bieber, C., \& Ruf, T. (2011). Hibernation is associated with increased survival and the evolution of slow life histories among mammals. Proceedings of the Royal Society B: Biological Sciences, 278(1723), 3355-3363. https://doi.org/10.1098/rspb.2011.0190

Turnbaugh, P. J., Bäckhed, F., Fulton, L., \& Gordon, J. I. (2008). Diet-Induced Obesity Is Linked to Marked but Reversible Alterations in the Mouse Distal Gut Microbiome. Cell Host \& Microbe, 3(4), 213-223. https://doi.org/10.1016/j.chom.2008.02.015

Turnbaugh, P. J., \& Gordon, J. I. (2009). The core gut microbiome, energy balance and obesity. The Journal of Physiology, 587(17), 4153-4158. https://doi.org/10.1113/jphysiol.2009.174136

Turnbaugh, P. J., Hamady, M., Yatsunenko, T., Cantarel, B. L., Duncan, A., Ley, R. E., Sogin, 
692 M. L., Jones, W. J., Roe, B. A., Affourtit, J. P., Egholm, M., Henrissat, B., Heath, A. C., Knight,

693 R., \& Gordon, J. I. (2009). A core gut microbiome in obese and lean twins. Nature, 457(7228),

694 480-484. https://doi.org/10.1038/nature07540

695

696 Turnbaugh, P. J., Ley, R. E., Mahowald, M. A., Magrini, V., Mardis, E. R., \& Gordon, J. I.

697 (2006). An obesity-associated gut microbiome with increased capacity for energy harvest.

698 Nature, 444(7122), 1027-1031. https://doi.org/10.1038/nature05414

Uenishi, G., Fujita, S., Ohashi, G., Kato, A., Yamauchi, S., Matsuzawa, T., \& Ushida, K. (2007).

701 Molecular analyses of the intestinal microbiota of chimpanzees in the wild and in captivity. American Journal of Primatology, 69(4), 367-376. https://doi.org/10.1002/ajp.20351

Vuren, D. V., \& Armitage, K. B. (2011). Duration of snow cover and its influence on life-history variation in yellow-bellied marmots. Canadian Journal of Zoology. https://doi.org/10.1139/z91$\underline{244}$

Weng, F. C.-H., Yang, Y.-J., \& Wang, D. (2016). Functional analysis for gut microbes of the brown tree frog (Polypedates megacephalus) in artificial hibernation. BMC Genomics, 17(13), 1024. https://doi.org/10.1186/s12864-016-3318-6

Wey, T. W., Lin, L., Patton, M. L., \& Blumstein, D. T. (2015). Stress hormone metabolites predict overwinter survival in yellow-bellied marmots. Acta Ethologica, 18(2), 181-185. Ludwig, W., \& Glöckner, F. O. (2014). The SILVA and "All-species Living Tree Project (LTP)" taxonomic frameworks. Nucleic Acids Research, 42(D1), D643-D648.

https://doi.org/10.1093/nar/gkt1209 


\section{$721 \quad$ Figures \& Tables}

722

723

724

725

726

727

728

729

730

731

Table 1. Characteristics of yellow-bellied marmots among selected samples $(N=201)$

\begin{tabular}{|l|c|c|c|c|c|}
\hline Characteristics & \multicolumn{5}{|c|}{ Number of samples (unique individuals) } \\
\hline Year & 2015 & 2016 & 2017 & 2018 & 2019 \\
\hline Age Class & \multicolumn{5}{|c|}{} \\
\hline Adult & $11(6)$ & $13(8)$ & $26(13)$ & $22(11)$ & $37(20)$ \\
\hline Yearling & $4(3)$ & $20(11)$ & $8(4)$ & $9(5)$ & $26(14)$ \\
\hline Juvenile & $2(1)$ & $3(2)$ & $7(4)$ & 0 & $13(7)$ \\
\hline Valley Position & \multicolumn{5}{|c|}{} \\
\hline Up-Valley & $16(9)$ & $29(16)$ & $30(15)$ & $20(10)$ & $27(14)$ \\
\hline Down-Valley & $1(1)$ & $7(5)$ & $11(6)$ & $11(6)$ & $49(27)$ \\
\hline
\end{tabular}




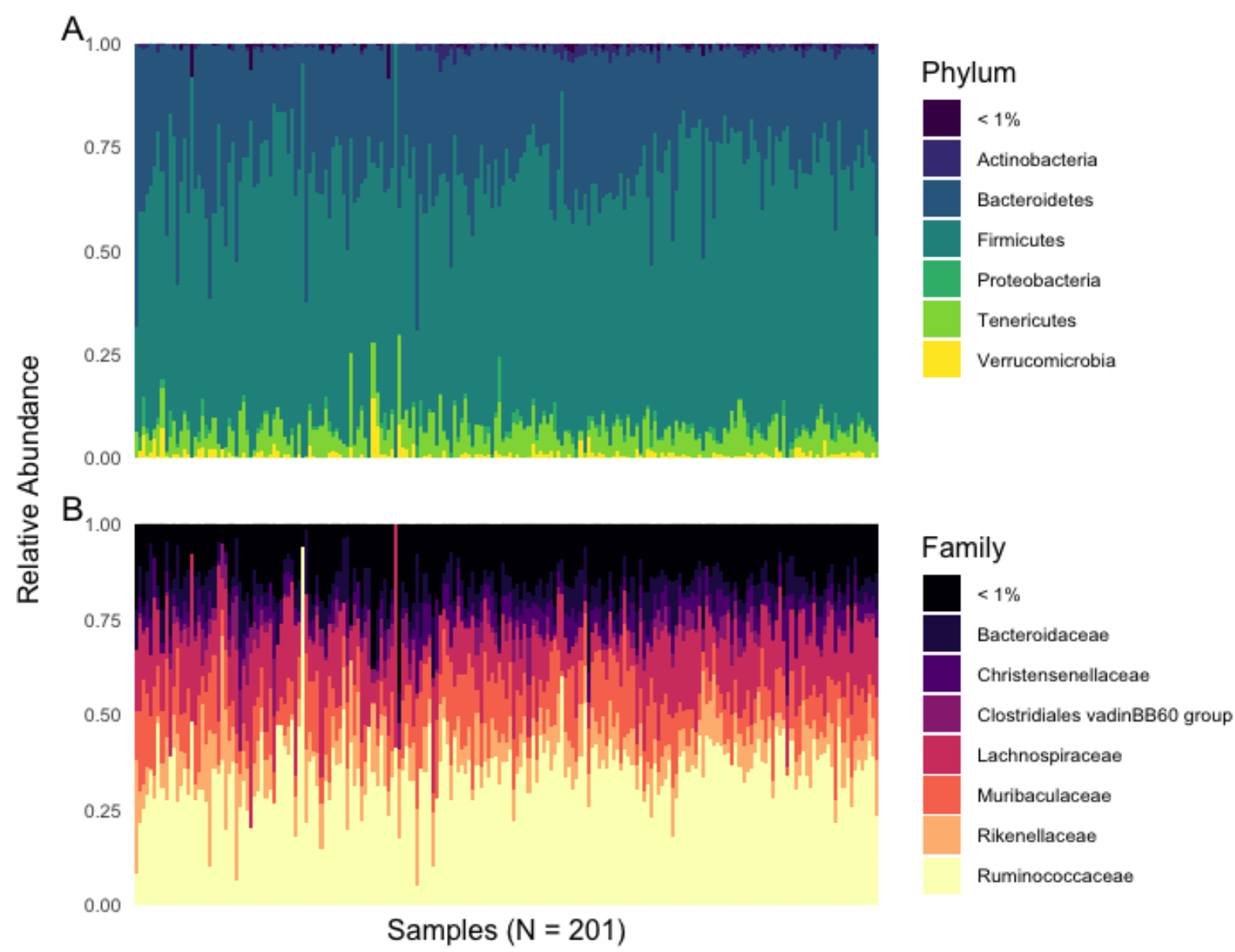

Figure 1. A) The relative abundance of dominant gut phyla across all samples $(N=201)$ showing Firmicutes and Bacteroidetes occupying the majority of reads. B) The relative abundance of dominant gut families across all samples $(N=201)$. 
A

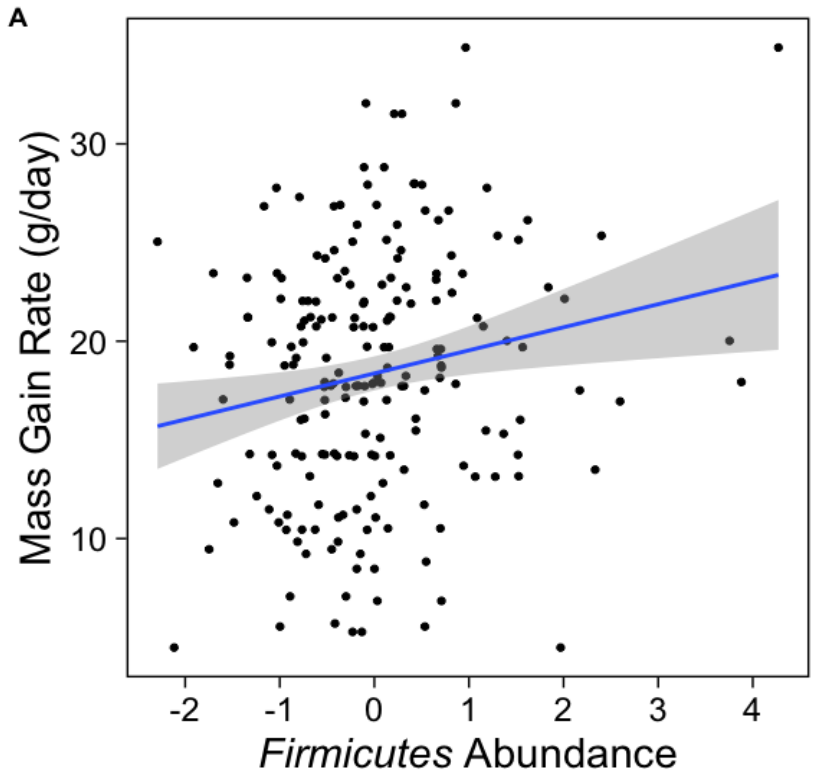

B

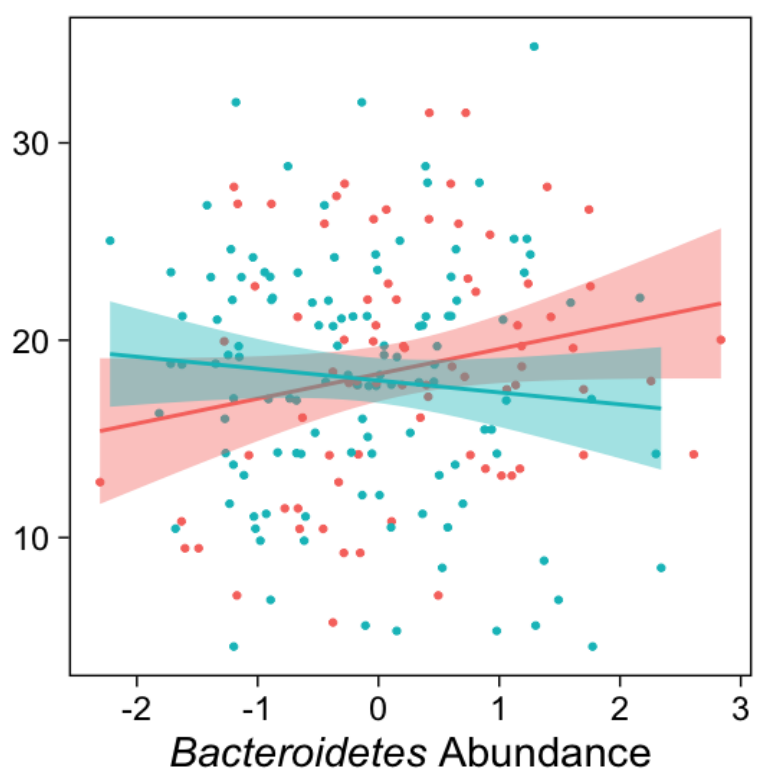

Figure 2. A) Relationship between Firmicutes abundance and mass gain rate for all samples $(N=$ 201) across the marmot active season. The blue line shows the predicted relationship based on the linear mixed effects model. B) Relationship between Bacteroidetes abundance and mass gain rate for all samples $(N=201)$ across the marmot active season. The blue line shows the predicted relationship based on the linear mixed effects model between Bacteroidetes abundance mass gain rate in higher elevation colonies, while the red line shows the predicted relationship between Bacteroidetes abundance and mass gain rate in lower elevation colonies. 
A

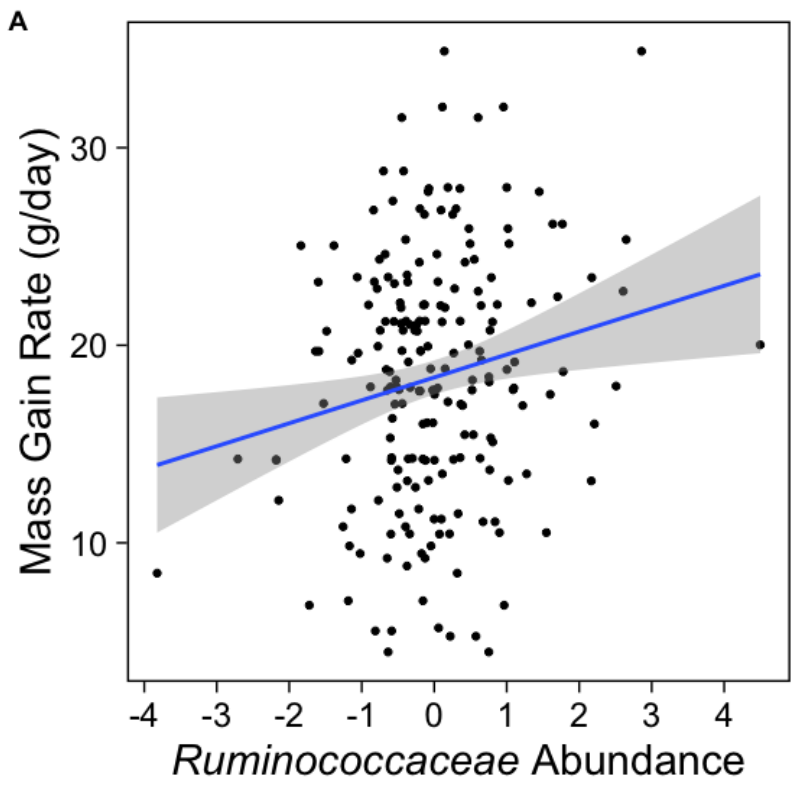

B

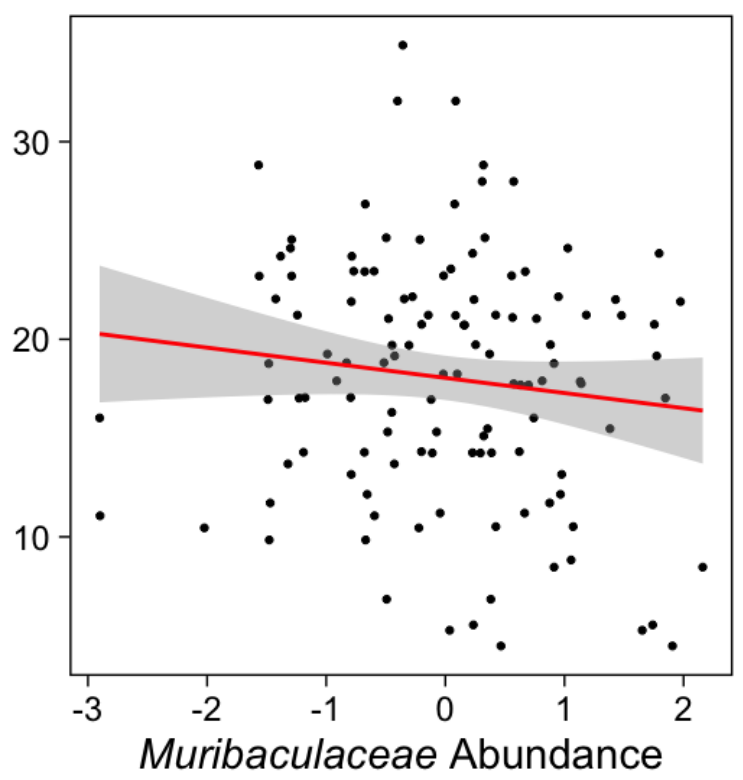

Figure 3. A) Relationship between Ruminococcaceae abundance and mass gain rate for all samples $(N=201)$ across the marmot active season. The blue line shows the predicted relationship based on the linear mixed effects model. B) Relationship between Muribaculaceae abundance and mass gain rate for only up valley samples $(N=122)$ across the marmot active season. The blue line shows the predicted relationship based on the linear mixed effects model between Muribaculaceae abundance mass gain rate in higher elevation colonies. 
Table 2. Fixed and random effects from the best fit model showing Firmicutes and age class explain variation in mass gain rates. The adult age class is used as the reference category. Significant effects are shown in bold.

788

Variable Estimate (SE)

$t$

$P$

(Intercept)

14.693

0.645

10.107

$1.9 \mathrm{e}^{-07}$

790

Firmicutes

$$
1.601
$$

2.712

0.007

791 Valley Position

$-3.505$

1.437

0.155

792 Age Class (J)

4.499

$-3.101$

0.002

793 Age Class (Y)

6.749

$1.67 \mathrm{e}^{-10}$

794 Random Effects

795

Groups Name

Variance

Std. Dev.

Individual UID

18.229

4.270

797

Year

5.464

2.337

Observations

201

799

Marginal $R^{2} /$ Conditional $R^{2}$

$0.175 / 0.831$

800

801

802

803

804

805

806

807

808

809

810

811

812

813

814

815

816

817

818

819

820

821

822 
823 Table 3. Fixed and random effects from the best fit model showing Bacteroidetes in up valley

824 colonies (UV) and age class explaining variation in mass gain rates. The adult age class is used

825 as the reference category. Significant effects are shown in bold.

$\begin{array}{llccc}826 & \text { Variable } & \text { Estimate }(\mathrm{SE}) & t & P \\ 827 & \text { (Intercept) } & \mathbf{1 5 . 0 0 8} & \mathbf{1 0 . 6 7 5} & \mathbf{3 . 9 8 e}^{-\mathbf{0 8}} \\ 828 & \text { Bacteroidetes } & 0.092 & 0.293 & 0.770 \\ 829 & \text { Valley Position (UV) } & 1.167 & 1.052 & 0.296 \\ 830 & \text { Age Class }(\mathbf{J}) & \mathbf{- 3 . 1 5 8} & \mathbf{- 2 . 7 6 3} & \mathbf{0 . 0 0 6} \\ 831 & \text { Age Class }(\mathbf{Y}) & \mathbf{4 . 3 8 0} & \mathbf{6 . 5 8 1} & \mathbf{4 . 2 4 e ^ { - 1 0 }} \\ 832 & \text { Bacteroidetes }^{-\mathbf{U V}} & \mathbf{- 0 . 9 7 5} & \mathbf{- 2 . 2 5 3} & \mathbf{0 . 0 2 6}\end{array}$

833 Random Effects

\begin{tabular}{lllc}
834 & Groups Name & Variance & Std. Dev. \\
\cline { 2 - 4 } 835 & Individual UID & 18.406 & 4.290 \\
836 & Year & 4.878 & 2.209 \\
837 & Observations & 201 & \\
838 & Marginal $R^{2} /$ Conditional $R^{2}$ & $0.172 / 0.829$ & \\
\hline
\end{tabular}


853 Table 4. Fixed and random effects from the best fit model showing Ruminococcaceae and age

854 class explain variation in mass gain rates. The adult age class is used as the reference category.

855 Significant effects are shown in bold.

856

Variable Estimate (SE)

$t$

(Intercept)

\subsection{9}

10.017

$4.68 \mathrm{e}^{-08}$

858

Ruminococcaceae

0.528

2.245

0.016

859 Valley Position

1.265

1.040

0.301

860 Age Class (J)

5.835

4.956

$1.68 \mathrm{e}^{-06}$

861 Age Class (Y)

4.284

6.293

$2.00 \mathrm{e}^{-09}$

862 Random Effects

863

Groups Name

Variance

Std. Dev.

864

Individual UID

18.550

4.307

865

Year

5.079

2.466

867 Marginal $R^{2} /$ Conditional $R^{2}$

$0.170 / 0.830$ 
890 Table 5. Fixed and random effects from the best fit model showing Muribaculaceae in up valley

891 colonies (UV) and age class explaining variation in mass gain rates. The adult age class is used

892 as the reference category. Significant effects are shown in bold.

893 Variable Estimate (SE) $t$

\begin{tabular}{|c|c|c|c|c|}
\hline 894 & (Intercept) & 15.201 & 10.286 & $2.62 \mathrm{e}^{-08}$ \\
\hline 895 & Muribaculaceae & -0.059 & -0.242 & 0.809 \\
\hline 896 & Valley Position (UV) & 1.123 & 0.933 & 0.353 \\
\hline 897 & Age Class (J) & 6.098 & 5.182 & $6.02 \mathrm{e}^{-07}$ \\
\hline 898 & Age Class (Y) & 4.357 & 6.445 & $8.88 e^{-10}$ \\
\hline 899 & Muribaculaceae $^{*} \mathbf{U V}$ & -1.416 & -2.667 & 0.008 \\
\hline
\end{tabular}

900 Random Effects

901 Groups Name Variance Std. Dev.

902 Individual UID $\quad 18.361$

$\begin{array}{llll}903 & \text { Year } & 4.928 & 2.446\end{array}$

904 Observations 201

905 Marginal $R^{2} /$ Conditional $R^{2} \quad 0.177 / 0.831$ 